\title{
Retinal detachment in identical twins with Stickler syndrome type 1
}

\author{
Yoshihiro Watanabe, Masahiro Ueda, Emiko Adachi-Usami
}

\begin{abstract}
Background-The high incidence of retinal detachment and its poor surgical prognosis in patients with Stickler syndrome are well known. However, the vitreoretinal relation to retinal detachment in this syndrome is uncertain.

Methods-Vitreoretinal examination with a binocular indirect ophthalmoscope and a Goldmann three mirror contact lens was performed on identical twin boys with Stickler syndrome. Each had retinal detachment in the left eye, and many aspects of their fundus findings were similar. The vitreous showed distinct abnormalities consistent with congenital vitreous anomaly of type 1 Stickler syndrome. The twins were followed up for 4 years after undergoing a successful operation to reattach their left retinas.
\end{abstract}

Results-Rhegmatogenous detachment with multiple tears occurred in the right eye of only one twin during the follow up period, despite the similar condition of their fundi. Although vitreous body was not present in most parts, slightly opaque vitreous cortex was attached to the retina near the ora serrata, and neither twin had posterior vitreous detachment during the follow up period.

Conclusion-Multiple retinal tears appeared simultaneously in the right eye of one twin, indicating some tractional force had acted on the retina. It is believed that this force was caused by very thin vestigial vitreous cortex attached to the retina. Although these observations have been limited, vitreoretinal findings of the twins and their father were consistent and suggested presence of thin vitreous cortex attached to the retina without posterior vitreous detachment.

(Br F Ophthalmol 1996;80:976-981)

Department of Ophthalmology, Chiba University School of Medicine, Japan Y Watanabe $M$ Ueda

E Adachi-Usami

Correspondence to: Yoshihiro Watanabe, MD, Department of Ophthalmology, Chiba University School of Medicine, 1-8-1 Inohana, Chuo-ku, Chiba 260, Japan.

Accepted for publication 1 August 1996 high incidence of retinal break formation in Stickler syndrome has not been explained with regard to the vitreoretinal relation. We recently treated retinal detachment in identical twins with Stickler syndrome and compared their fundi in detail during the 4 year follow up.

\section{Materials and methods}

Identical twin boys with Stickler syndrome referred for retinal detachment in their left eyes were examined by indirect ophthalmoscopy with scleral depression and fundus biomicroscopy with a Goldmann three mirror contact lens. All fundus lesions in both twins were recorded precisely by drawing and photography. The boys were followed up for 4 years, after undergoing a successful operation to reattach their left retinas, to compare the prognosis of their condition.

\section{Results}

CASE 1

On 1 May 1990, a 12-year-old boy who was an identical twin was referred to us for retinal detachment in his left eye. The patient reported that his visual loss had started in October 1989 and gradually progressed. Corrected visual acuity was $20 / 20$ in the right eye and 20/100 in the left eye. Slit-lamp examination revealed optically empty vitreous with a few floating strands attached to the retina in both eyes. There was no discernible collagen structure and only a few filmy mobile membranes in the retrolental space. These findings were consistent with congenital vitreous anomaly described by Scott. ${ }^{3}$ The detached retina in the left eye extended clockwise from $2: 30$ to $8: 30$. Two small equatorial retinal breaks were seen at 2:30, and two peripheral breaks were noted at 3 o'clock. Some small holes existed in areas of lattice degeneration at 2:30 and 3:30. Lesions of pigment epithelial thinning were seen at the superior equator. Bands of thin retina, which presumably corresponded to areas of pigment epithelial thinning before retinal detachment occurred, ran along the retinal vessels at the inferior equator (Fig 1). In the right eye were several areas of thinned pigment epithelium at the equator and a 180 degree 'white without pressure' at the temporal periphery (Fig 2). In both eyes, thin vitreous veils were attached to the retina in several areas. No glial ring or posterior hyaloid membrane was seen on biomicroscopy with a Goldmann three mirror contact lens.

Based on these biomicroscopic findings, we believed that very thin vitreous cortex was still attached to the retina without posterior vitreous detachment (PVD). We successfully repaired the left retinal detachment with cryopexy, drainage of subretinal fluid, and encircling with an episcleral buckle. The 


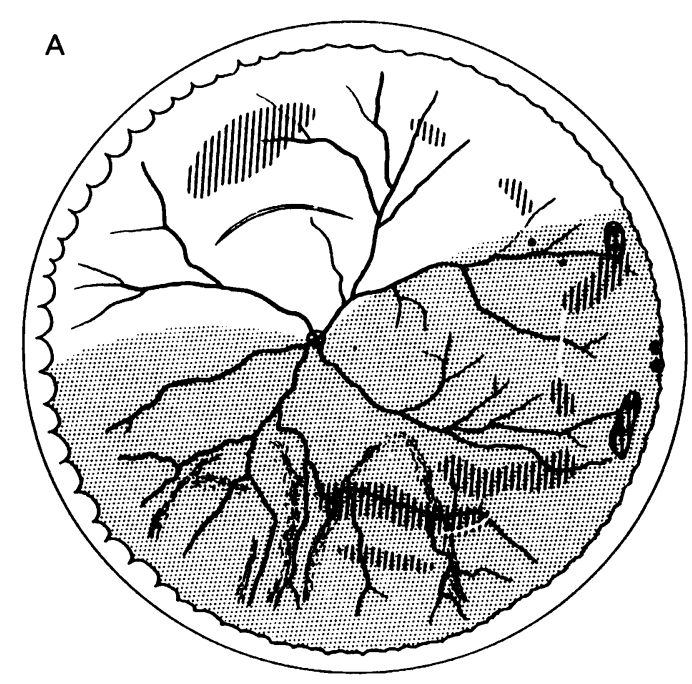

Figure 1 (A) Left fundus of case 1 on 12 May 1990 (arteries not shown.) Retina was detached clockwise from 2:30 to 8:30. Two small equatorial retinal breaks are seen at 2:30, and two peripheral breaks are noted at $3 o^{\prime}$ 'clock. Some small holes exist in areas of lattice degeneration at 2:30 and 3:30. Pigment epithelial thinning occurs in the superior equator. Bands of thin retina, which are presumed to have corresponded to areas of pigment epithelial thinning before retinal detachment occurred, run along the retinal vessels at the inferior equator. Three vitreous veils are attached to retina in the equatorial region. (B) Photomontage of the left fundus of case 1.
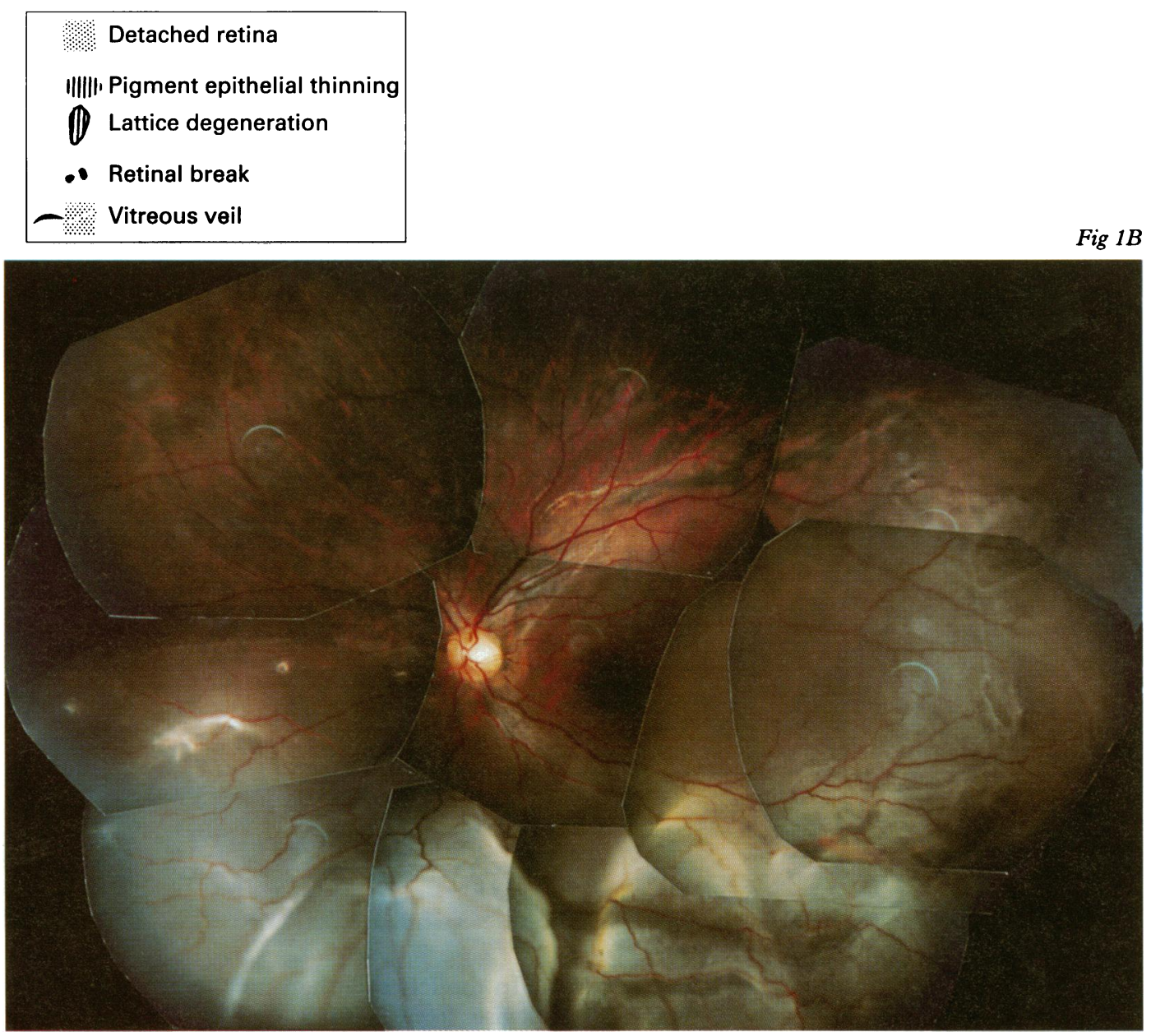

refractive error based on retinoscopy after reattachment of the left retina was $-4.0-0.5 \times 3$ in the right eye and $-6.5-0.25 \times 160$ in the left eye. On 24 September 1990, the boy noticed blurred vision and visual field constriction in his right eye. Funduscopic examination showed multiple retinal tears in the temporal equator and total cystic retinal detachment (Fig 3). The breaks at 10 o'clock corresponded to those areas of pigment epithelial thinning noted previously. Fundus biomicroscopy revealed condensed cortical vitreous attached to the retina near the ora serrata at the temporal periphery. Vitreous veils were still attached to the retina, without PVD. Despite scleral buck- ling and repeated vitreous surgery for proliferative vitreoretinopathy (PVR), we could not reattach the retina.

CASE 2

The twin brother of case 1 was referred to us for retinal detachment in his left eye on 1 May 1990 , the same day his twin was initially evaluated. He had been aware of left visual field constriction from the superonasal direction since April 1989, but his parents thought his visual loss was caused by myopia and did not consult any doctor until later. Corrected visual acuity was right eye $20 / 20$ and left eye $20 / 40$. The vitreous showed the same congenital 


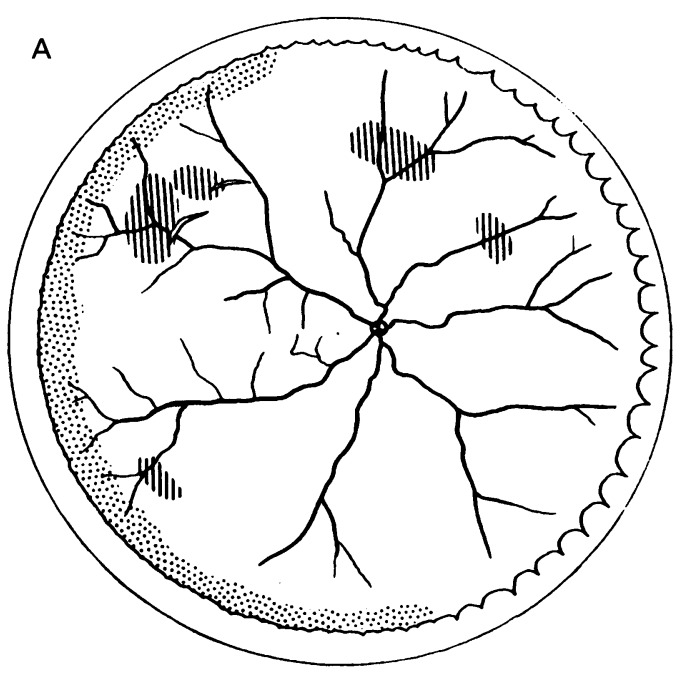

Figure 2 (A) Right fundus of case 1 on 12 May 1990. Several areas of thinned pigment epithelium are seen at the equator and a 180 degree 'white without pressure' is observed at the temporal periphery. (B) Photomontage of the right fundus of case 1. Vitreous veils are attached to the areas of pigment epithelial thinning at the superotemporal equator.

$$
\begin{aligned}
& \|\|\|\| \text { Pigment epithelial thinning } \\
& \text { : White without pressure } \\
& \subset \text { Vitreous veil }
\end{aligned}
$$

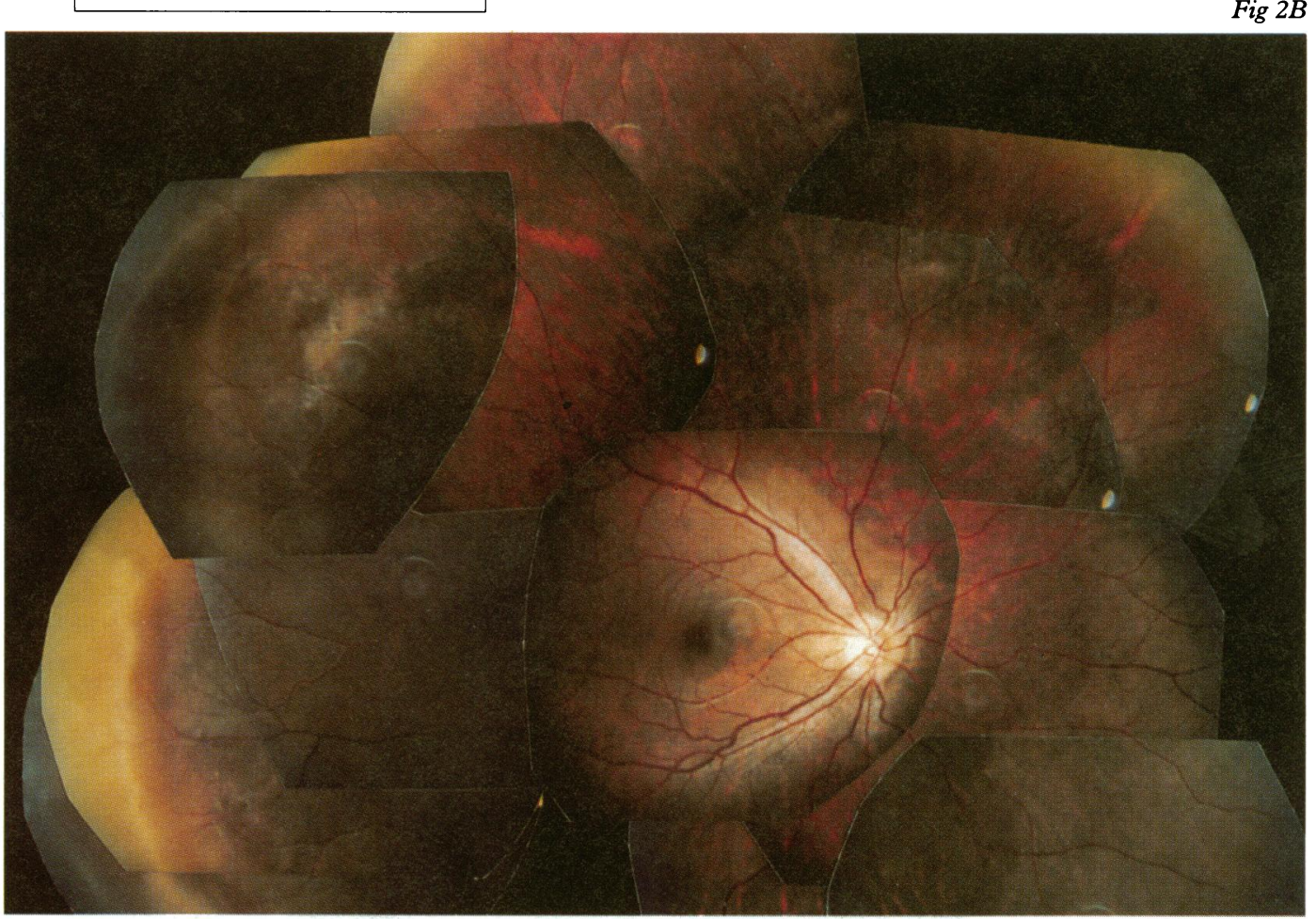

anomaly as seen in case 1 . In the left eye, the retina was detached clockwise from 3 to 7 o'clock. One small retinal break was seen at 4 o'clock near the ora serrata, and small holes were found in areas of lattice degeneration at 3 o'clock and in a band of thin retina in the inferior quadrant. This band of thin retina ran along the retinal vessels and was presumed to correspond to areas of pigment epithelial thinning before the retinal detachment had occurred (Fig 4). In the right eye, areas of thinned pigment epithelium ran circumferentially near the equator and along the retinal vessels posteriorly at the superotemporal quadrant. 'White without pressure' extended from the temporal to the inferior periphery (Fig 5). Fundus biomicroscopy with use of a Goldmann lens revealed thin vitreous veils attached to the retina in several areas and no PVD in either eye.

We successfully repaired the left retinal detachment with cryopexy, drainage of subretinal fluid, and encircling with an episcleral buckle. The refractive error based on retinoscopy after reattachment of the left retina was $-7.0-0.75 \times 180$ in the right eye and -10.0 $-0.75 \times 180$ in the left eye. There was no recurrence during the follow up period. His final left visual acuity was $20 / 30$.

The twins underwent the repair of cleft palate when they were 1 year old, and their $x$ ray findings showed dysplasia of hip, knee, and ankle joints. Ocular findings, their past medical history, $x$ ray findings, and family medical history were consistent with the diagnosis of Stickler syndrome. 

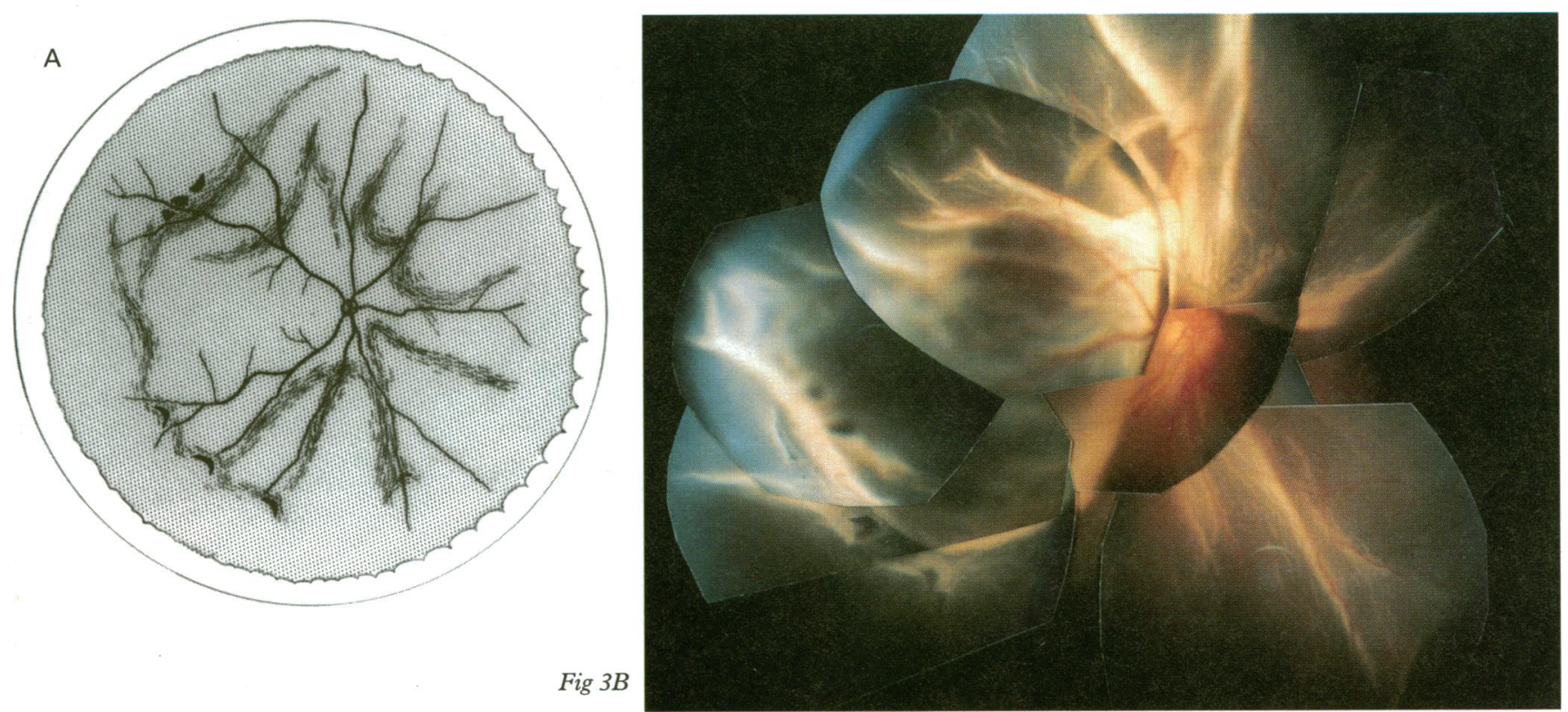

Fig $3 B$

Figure 3 (A) Right fundus of case 1 on 2 October 1990, with patient in the supine position. Six retinal tears were found at the temporal equator. Most correspond to areas of pigment epithelial thinning seen before retinal detachment occurred, but some tears appeared elsewhere, where vitreoretinal adhesion had not been visible. (B) Photomontage of right retinal detachment of case 1 with patient in a sitting position. Superotemporal breaks shifted inferiorly because of the bullous detachment.

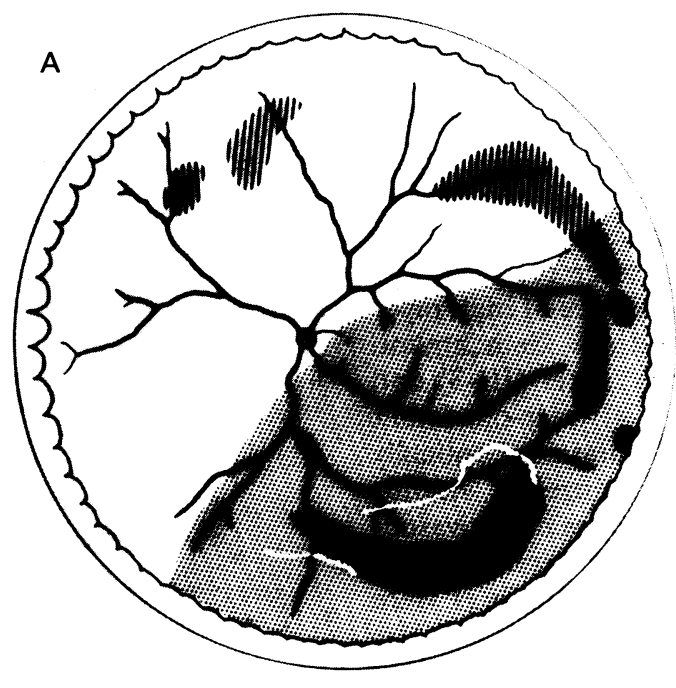

Figure 4 (A) Left fundus of case 2 on 12 May 1990. Inferotemporal retinal detachment occurs with a break near the ora serrata and holes in areas of thinned pigment epithelium and lattice degeneration. Vitreous veils are attached to the retina at the inferior equator.

(B) Photomontage of the left fundus of case 2.

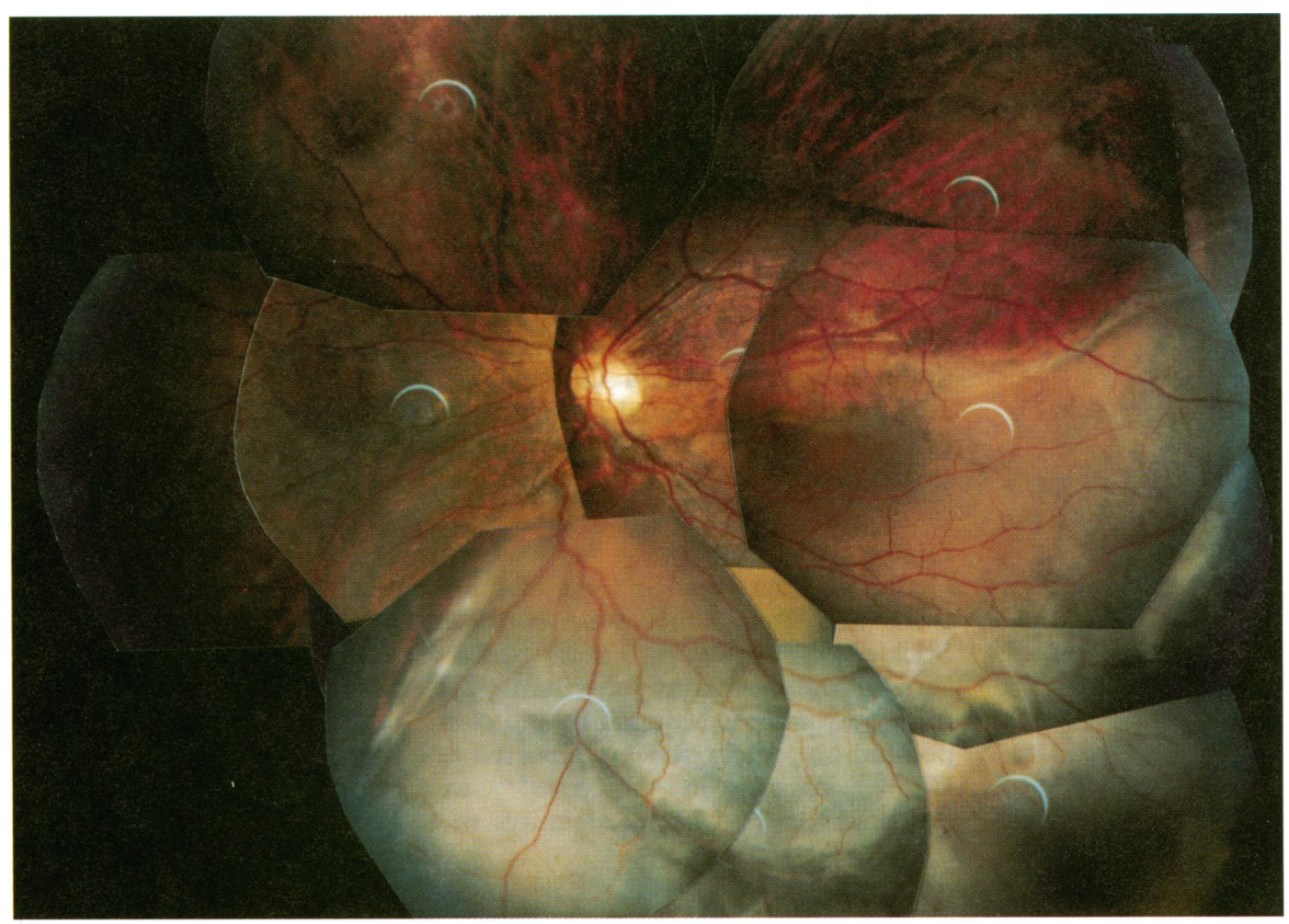



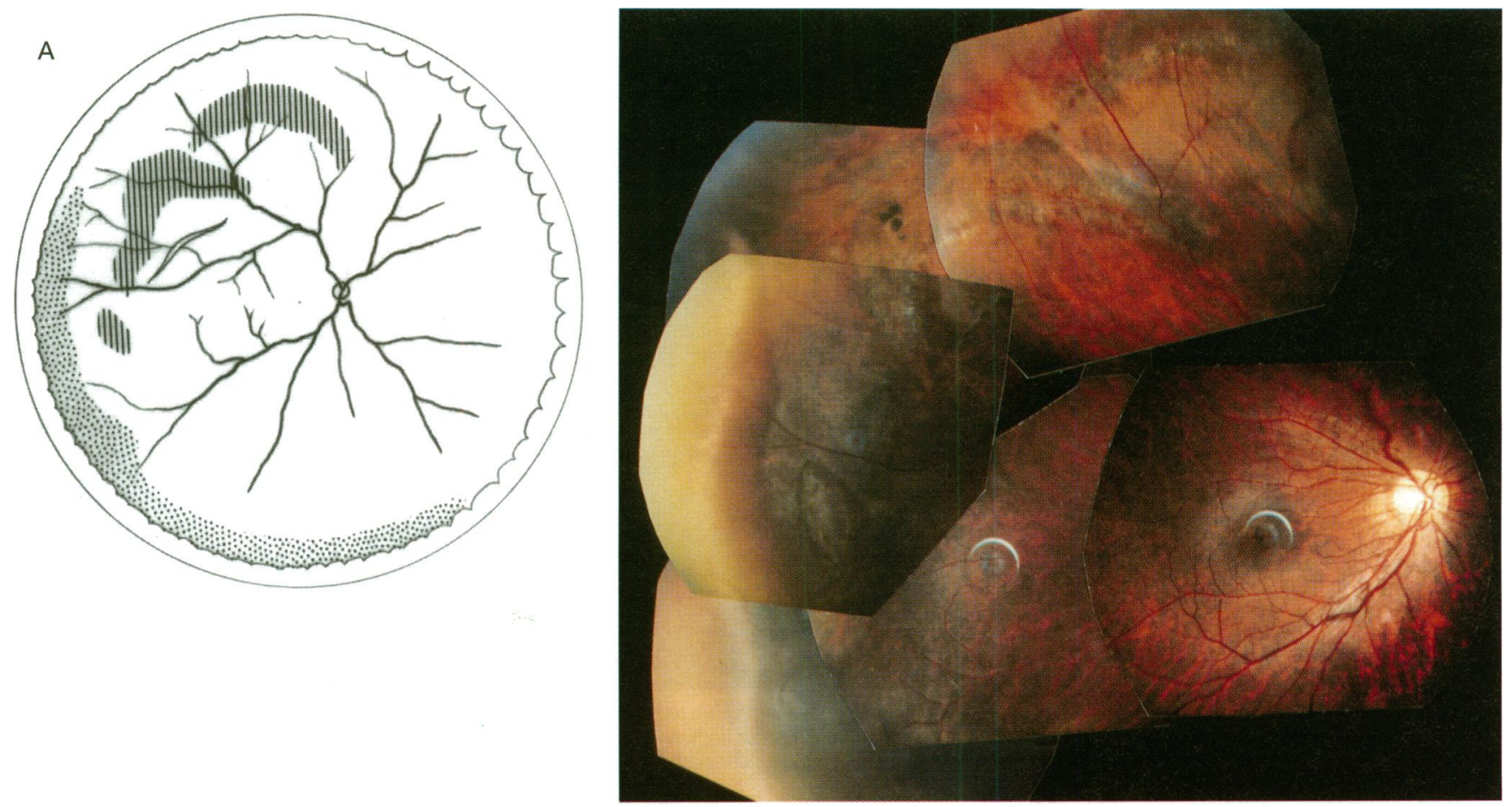

Fig $5 B$

Figure 5 (A) Right fundus of case 2 on 12 May 1990. Several areas of thinned pigment epithelium are seen at the equator and a 180 degree 'white without pressure' is noted at the inferotemporal periphery. Vitreous veils are attached to the retina at the superotemporal equator. The fundus appearance is similar to that seen in Figure 2. (B) Photomontage of the right fundus of case 2.

FAMILY HISTORY

We examined the parents and the sister of the twins, and gained information about their pedigree from the twin's father (Fig 6). The pedigree showed a high incidence of high myopia, cleft palate, and retinal detachment. Ocular findings of the twins' sister (III-3) and mother were normal. Their 47-year-old father (II-3) showed presenile cataract, optically empty vitreous with some vitreous strands attached to the retina, many areas of perivascular chorioretinal atrophy with pigmentation, and micrognathia. His vitreous findings were fundamentally the same as his twin sons. The father underwent cataract surgery of the right eye on 27 August 1991, and his best corrected visual acuity was $20 / 30$ postoperatively.

\section{RETINAL FUNCTION}

The bright flash electroretinogram (ERG) was recorded in five members of this family (III-1, III-2, III-3, and their parents). The ERG was normal in the mother and the sister of the twins, but the amplitude of the oscillatory potentials was decreased in the twins and was almost extinguished in their father. Dark adaptation measured with a Goldmann-Weekers adaptometer was normal (5 logarithmic units of change) in this family except for the father (lower than 4 logarithmic units of change).

\section{Discussion}

Ocular and systemic findings of the identical twins and their father were consistent with Stickler syndrome. ${ }^{4-7}$ Their pedigree also showed the dominant inheritance pattern of this syndrome. As Maumenee and coworkers ${ }^{89}$ pointed out, many patients reported as having Wagner disease in the past $^{1011}$ were not screened for the systemic features of Stickler

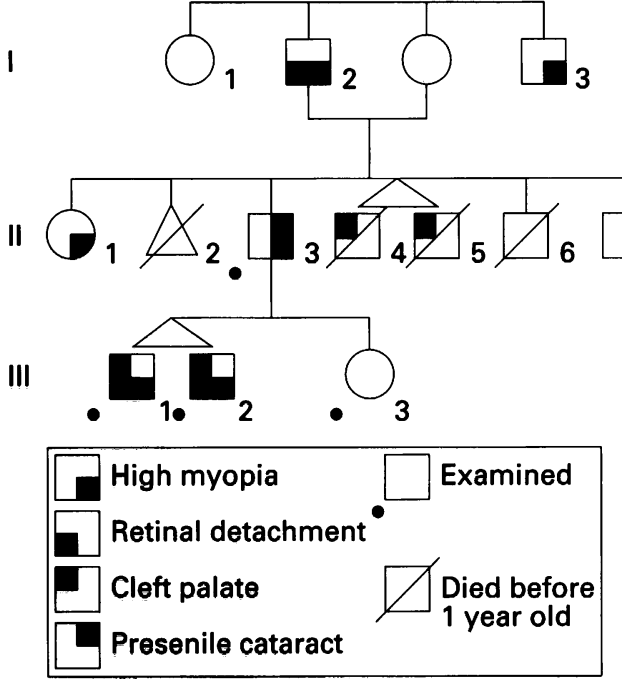

Figure 6 Pedigree of affected family. The probands are III-1 and III-2. Twin uncles of the reported twins (II-4 and II-5) died 10 days after birth. They were unable to suck, and were presumed to have had cleft palate. The

grandfather of the reported twins (I-2) lost visual acuity in his right eye before age 40 years without an exogenous condition. He was presumed to have had retinal detachment.

syndrome, and their condition may have been more properly categorised as Stickler syndrome. Decreased oscillatory potentials of ERGs in our patients also coincided with subnormal ERGs reported previously. ${ }^{10}$ Formerly, a vitreous condition like that seen in our cases was described as vitreous liquefaction. More recently, it has been known that normal vitreous structures are congenitally absent, and the condition should have been expressed as vitreous anomaly. ${ }^{3}$ Snead et al ${ }^{12}$ recently classified Stickler syndrome into type 1, with retrolental vitreous anomaly, and type 2 , without retrolental anomaly, and demonstrated 
linkage to $\mathrm{COL} 2 \mathrm{~A} 1$ in type 1 pedigrees. Because our cases showed retrolental vitreous anomaly, we diagnosed them as having Stickler syndrome type 1 .

Stickler syndrome has a high incidence of retinal detachment and a low success rate of surgery. ${ }^{11}$ The reasons for poor surgical results are presumed to be multiple retinal breaks located at different distances from the limbus, ${ }^{10}$ but whether these breaks are caused by PVD or by some exogenous factor is uncertain.

In our cases, the retinal detachments that occurred in the left eyes of both twins and in the right eye of case 1 were different in many aspects. Demarcation lines with pigmentation seen in the left eyes (Figs 1 and 4) showed chronicity. These retinal detachments were mainly caused by atrophic retinal holes that occurred in the area of lattice degeneration or pigment epithelial thinning, probably without rupture of thin vitreous cortex attached to the retina. Subsequently, vitreous collapse did not occur, and retinal detachments progressed slowly. On the other hand, in the right eye of case 1 , multiple retinal tears led to total cystic retinal detachment within a few days, which indicated vitreous collapse. The biomicroscopic findings showed that this vitreous collapse was not accompanied by PVD but was caused by multiple tears of vitreous cortex that developed simultaneously with the multiple retinal tears at the areas of vitreoretinal adhesion. In Stickler syndrome type 1, almost all vitreous is congenitally absent and determining whether PVD is present or not is very difficult. However, we believed that PVD was not present, because vitreous strands attached to the detached retina posterior to the retinal breaks were observed, and no glial ring or posterior hyaloid membrane was seen in the left eyes of the twins or in the right eye of case 1 by biomicroscopy with a Goldmann contact lens.

Because identical twins have no genetic differences, comparing their clinical appearance is important to assess environmental factors. Nordlow ${ }^{1}$ reported on retinal detachment in the right eyes of identical twins, and Verdaguer et $a l^{2}$ described retinal detachments caused by dialysis in the contralateral eyes of twins. However, it was uncertain whether these twins had hereditary retinal detachment. Funduscopic findings before retinal detachment developed were not described. Although the position of 'white without pressure' and pigment epithelial thinning were also alike in the right eyes of our twins, case 1 developed bullous retinal detachment within 3 months after the first examination and case 2 showed no change during the 4 year follow up period. The different clinical courses in these eyes suggest some environmental factor as the cause of the detachment, because both eyes should have had the same risk factors.

Based on the clinical findings from our case studies, we think that the reason for frequent retinal detachment in Stickler syndrome is as follows. People with Stickler syndrome have two inherited ophthalmic risk factors. Firstly, many areas of the retina are vulnerable with associated lattice degeneration and pigment epithelial thinning. Secondly, almost all vitreous body is congenitally absent, but the sheet of condensed vitreous cortex is firmly attached to the vulnerable areas of the retina. If atrophic retinal breaks develop in these areas but the sheet of vitreous cortex remains intact, retinal detachment will progress slowly, as in the left eyes of our twins. On the other hand, if simultaneous rupture of the retina and the vitreous cortex occurs at the vulnerable areas, liquefied vitreous will pour into the subretinal space to produce bullous retinal detachment rapidly. Furthermore, vitreous cortex still attached to the retina after vitreous collapse will provide scaffolding for cell proliferation and an environment for PVR.

1 Nordlow W. Fall von spontaneous Netzhautablosung bei eineiigen Zwilligen. Acta Ophthalmol 1938;16:579-88.

2 Verdaguer TJ, Rojas B, and Lechuga M. Genetical studies in nontraumatic retinal detachment. Mod Probl Ophthalmol 1975;15:34-9.

3 Scott JD. Congenital myopia and retinal detachment. Trans Ophthalmol Soc UK 1980;100:69-71.

4 Stickler GB, Belau PG, Farrell GJ, Jones JD, Pugh DG, Steinberg AG, et al. Hereditary progressive arthroophthalmopathy. Mayo Clin Proc 1965;40:433-55.

5 Stickler GB, Pugh DG. Hereditary progressive arthroophthalmopathy. II. Additional observations on vertebral abnormalities, a hearing defect, and a report of a similar case. Mayo Clin Proc 1967;42:495-500.

6 Blair NP, Albert DM, Liberfarb RM, Hirose T. Hereditary progressive arthro-ophthalmopathy of Stickler. Am $\mathcal{F} O \mathrm{ph}$ thalmol 1979;88:876-88.

7 Temple IK. Stickler's syndrome. F Med Genet 1989;26:119-

8 Maumenee IH, Stoll HU, Metz MB. The Wagner syndrome vs hereditary arthro-ophthalmopathy. Trans Am Ophthalmol Soc 1982;80:349-65.

9 Maumenee IH. Vitreoretinal degeneration as a sign of generalized connective tissue disease. Am $\mathcal{f}$ Ophthalmol 1979;88:432-49.

10 Hirose T, Lee KY, Schepens CL. Wagner's hereditary vitreoretinal degeneration and retinal detachment. Arch Ophthalmol 1973;89:176-85.

11 Brown GC, Tasman WS. Vitrectomy and Wagner's vitreoretinal degeneration. Am $\mathcal{f}$ Ophthalmol 1978;86:485-8.

12 Snead MP, Payne SJ, Barton DE, Yates JW, Al-Imara L, Pope FM, et al. Stickler syndrome: correlation between vitreoretinal phenotypes and linkage to COL 2A1. Eye 1994; 8:609-14. 